\title{
Editorial
}

\section{Dipeptidyl peptidases and related proteins: multifaceted markers and therapeutic targets}

By far the best known member of this family of peptidases is dipeptidyl peptidase 4 (DPP4, DPPIV, EC 3.4.14.5), an enzyme that was discovered more than four decades ago. It is expressed in a variety of tissues and a soluble form is present in plasma. It was found to be identical to the cell surface protein CD26. Complementary research in clinical chemistry, enzymology and immunology initially investigated the diagnostic and prognostic value of CD26/DPP4 (1). No prominent diagnostic value emerged (so far) for the soluble plasma DPP4, although methods for plasma DPP4 activity measurements are available since the 1980s. On the other hand, cell surface expression of DPP4 repeatedly is proposed as a marker for differentiated thyroid carcinoma (2). Within the circulating lymphocyte population, the CD26 bright (also called CD26 ${ }^{\text {high }}$ ) cells belong to the CD45R0 + population and represent a distinct functional subset of T-cells, which is responsible for the response to recall antigens, allogeneic cells and alloantigens. Monitoring CD26 bright lymphocyte levels is a promising tool during clinical follow-up in defined conditions, such as immune deficiency or in studies of immune responses to infectious agents or new vaccine candidates (3). Apart from that, CD26 bright cells seem to be enriched in the course of progenitor cell apheresis. High numbers of CD26 $6^{\text {bright }}$ cells in the autograft predict a shorter event-free survival. In this clinical situation, a depletion of CD26 ${ }^{\text {bright }}$ cells from the peripheral blood stem cell autograft might be considered (4).

In addition to the search towards the 'marker' value of DPP4/CD26, thorough enzymological and structural characterization of DPP4 during the 1990s allowed the development of potent inhibitors (5). The evaluation of inhibitors in several animal models of disease and the unexpectedly favorable animal safety profile forced a breakthrough in the development of DPP4 inhibitors as a new class of therapeutics.

The "3rd International Conference on Dipeptidyl peptidases (DPP) \& Related Proteins" was devoted to the emerging DPP family of proteases focusing on basic science, clinical issues and future perspectives. This conference, a follow-up of a series of successful meetings organized by Prof. Ansorge in Magdeburg (6) and Prof. Hildebrandt in Berlin (7) was held in Antwerp, April 2008 (8). The 'multifaceted' aspects of DPP4 and related proteins are illustrated in this issue by the contribution of $\mathrm{N}$. Frerker and colleagues on the integration of DPP4 in complex biological networks. In their papers, S. Ansorge and D. Reinhold show experimental evidence that some small molecular weight DPP4 inhibitors hit other targets and that this effect might contribute to their efficacy in certain preclinical pharmacology studies. Drug discovery efforts during the past two decades indeed resulted in the development of a vast amount of small molecular weight DPP4 inhibitors of distinct chemical structures and beneficial in vivo effects. Perhaps the time has come to look for additional targets of these compounds (9). The future will tell whether additional new classes of therapeutics are hidden in the present compound libraries and datasets. DPP10, a homolog of DPP enzymes, has been presented as a potential new target for asthma therapy (10). K. McNicholas and colleagues, in this issue, review recent data on the non-enzyme family members including not only DPP10 but also DPP6.

While a number of papers in this issue and elsewhere highlight future directions of DPP related research, we should also consider the clinical use of DPP inhibitors today in relation to laboratory medicine practice.

DPP 4 has become a target in the development of oral antidiabetics as soon as its role in the in vivo inactivation of the incretins glucagon-like-peptide 1 (GLP-1) and glucose dependent insulinotropic peptide became clear (11). The incretins are peptide hormones released in the intestine upon food intake. GLP-1 enhances insulin release in a glucose dependent manner, suppresses glucagon secretion and hence lowers blood glucose levels. Animal and in vitro studies also point to $\beta$-cell trophic actions of GLP-1. In humans, this effect has yet to be demonstrated (12). The in vivo half-life of GLP-1, however, is very short. The rapid inactivation occurs by $\mathrm{N}$-terminal truncation by DPP4 that cleaves off a dipeptide. Small molecule DPP4 inhibitors potentiate and prolong the effects of endogenous incretins. They are called 'incretin enhancers'. Long-acting stable analogs of GLP-1 also called 'incretin mimetics' have been introduced in diabetes treatment since 2005. Their parenteral administration is a drawback for application in early type 2 diabetes care. Two orally active DPP4 inhibitors are currently used in the treatment of type 2 diabetes: sitagliptin (Januvia ${ }^{\circledR}$ ) and vildagliptin (Galvus ${ }^{\circledR}$ ). Many more are in different phases of clinical research. Incretin enhancement certainly is the main mechanism of action of the DPP4 inhibitors but other effects may contribute. Based on the major mechanism of action, one can assume that DPP4 inhibitors will have the greatest effect when started early during the course of type 2 diabetes (11).

To date, two systematic reviews and meta-analyses of the efficacy and safety of DPP4 inhibitors in type 2 
diabetes have been published $(13,14)$. DPP4 inhibitors showed $\mathrm{HbA}_{1 \mathrm{c}}$ improvements of $0.7 \%$ vs. placebo in both meta-analyses. The available data suggest that they are slightly less efficient than other hypoglycemic agents. However, when combined with other antidiabetic compounds, both vildagliptin and sitagliptin lead to an additional lowering of $\mathrm{HbA}_{1 \mathrm{c}}$. Combination therapy of a DPP4 inhibitor with metformin (fixed in Eucreas ${ }^{\circledR}$ and Janumet ${ }^{\circledR} /$ Efficib $^{\circledR}$ ) or with a sulfonylurea is used when metformin and/or sulfonylurea alone proves to be insufficient for glycemic control. $\mathrm{HbA}_{1 \mathrm{c}}$ is the parameter to be followed during type 2 diabetes treatment with DPP4 inhibitors. There is currently no evidence for the use of plasma DPP4 activity measurement in this clinical setting. There are no clinically meaningful interactions of sitagliptin or vildagliptin with co-administered medications, such as oral anticoagulants, so far. Especially in the target population - type 2 diabetes patients - this is highly desirable.

Meta-analysis confirmed earlier reports on an overall low risk profile of DPP4 inhibitors and no major side effects were mentioned during therapy $(13,14)$. The withdrawal rate due to adverse effects was not significantly higher in the DPP4 inhibitor groups than in controls. The lack of major hypoglycemic events is considered as an important advantage for this new class of oral antidiabetics. Post-marketing, a few serious hypersensitivity reactions (including anaphylaxis, angioedema, rash and exfoliative skin conditions) have been reported upon use of sitagliptin. For vildagliptin, rare cases of generally asymptomatic hepatic dysfunction have been reported; they were without clinical sequellae upon drug withdrawal. After combining data from clinical studies, Richter et al. found an increased risk of all-cause infections for sitagliptin but not for vildagliptin (14). Amori et al. report a slightly increased risk of infection for vildagliptin also (13). The clinical significance is not completely clear at present, nor can one propose a clearly defined causative mechanism.

Apart from GLP-1, a number of other biologically active peptides are truncated by DPP4 $(1,15)$. For most of them, physiological relevance of DPP4 for their metabolism still has to be established. The lack of appropriate methodology to measure intact vs. truncated (i.e., minus two amino acids) bio-active peptides in vivo certainly is one of the main reasons for this incomplete understanding.

In the near future, methods based on combined immuno-affinity enrichment and mass spectrometrical detection might become available for various substrates. This will allow to indisputably and quantitatively measure differently processed forms of bioactive peptides. For B-type natriuretic peptide, the present insights in the in vivo processing are discussed further in this issue in the paper by $M$. Vanderheyden.

Evidence for the physiologically relevant truncation of another DPP4 substrate, CXCL12 or Stromal cell Derived Factor $1 \alpha$ comes from a recent case report on an improved engraftment of hematopoietic stem cell transplantation by DPP4 inhibition with sitagliptin (16).
Albeit not yet at the sensitivity level one would dream of in the area of clinically relevant bioactive peptides, the 'omics' approach allows researchers today to obtain a tissue/plasma wide peptide profile using multidimensional LC-mass spectrometry methodologies. Two recent independent studies confirm earlier hypotheses on the involvement of DPP4 in the metabolism of proline containing proteins and peptides $(17,18)$. DPP4 cleaves with a high efficiency peptides that have a proline on the penultimate position. Those peptides are formed to an appreciable amount, because 'regular' aminopeptidases cannot truncate a peptide just before a proline residue. As collagen is a proline-rich protein, it is not surprising that in vivo DPP4 inhibition significantly changes the profile of collagen derived peptides (17). Collagen is considered to be the proline reservoir of the body. Sequential actions of metalloproteinases, peptidases (e.g., DPP4) and prolidase can increase the free proline concentration. Whether the in vivo inhibition of individual steps within the proline mobilization process finally influences the free proline concentration and proline metabolism in certain clinical conditions remains to be investigated (19).

New analysis platforms, such as SELDI-TOF mass spectrometry, allow a further exploration of DPP4/ CD26 and the more recently discovered family members as biomarkers during disease or response to treatment. The discovery of DPP4 as a biomarker during follow-up of enzyme replacement therapy in mucopolysaccharidoses nicely illustrates that the DPPs and related proteins definitely entered the realm of clinical and laboratory medicine (20).

In conclusion, DPP inhibitors hold great promise in the treatment of diabetes and beyond. It is, however, somewhat premature to predict whether the success track of angiotensin converting enzyme from a diagnostic tool to a highly valuable therapeutic target will be repeated for DPP4.

\section{References}

1. Lambeir AM, Durinx C, Scharpé S, De Meester I. Dipeptidyl-peptidase IV from bench to bedside: an update on structural properties, functions, and clinical aspects of the enzyme DPP IV. Crit Rev Clin Lab Sci 2003;40:209-94.

2. de Micco C, Savchenko V, Giorgi R, Sebag F, Henry JF. Utility of malignancy markers in fine-needle aspiration cytology of thyroid nodules: comparison of Hector Battifora mesothelial antigen-1, thyroid peroxidase and dipeptidyl aminopeptidase IV. Br J Cancer 2008;98:81823.

3. Ibegbu CC, Xu YX, Fillos D, Radziewicz H, Grakoui A, Kourtis AP. Differential expression of CD26 on virus-specific $\mathrm{CD} 8(+) \mathrm{T}$ cells during active, latent and resolved infection. Immunology 2008 (E-pub ahead of print) doi 10.1111/j.1365-2567.2008.02899.

4. Hildebrandt $H$, Gollasch $H$, Rautenberg $K$, Ludwig W-D, Dörken B. The levels of CD26 $6^{\text {positive }}$ lymphocytes and CD26 bright/CD45RO pos memory T-cells in the autograft predict early progression or recurrence in patients undergoing high-dose chemotherapy and autologous haematopoietic progenitor-cell transplantation. Clin Chem Lab Med 2008;46:A17. 
5. Van der Veken P, Haemers A, Augustyns K. Prolyl peptidases related to dipeptidyl peptidase IV: potential of specific inhibitors in drug discovery. Curr Top Med Chem 2007;7:621-35.

6. Lendeckel U, Bank U, Reinhold D, editors. Dipeptidyl aminopeptidases: basic science and clinical applications. New York: Springer, 2006:239 pp.

7. Hildebrandt M, Klapp B, Hoffmann T, Demuth H-U, editors. Dipeptidyl aminopeptidases in health and disease. New York: Kluwer Academic/Plenum Publishers, 2003: $359 \mathrm{pp}$.

8. All authors. Abstracts 3rd International Conference on Dipeptidyl Peptidase and Related Proteins. Clin Chem Lab Med 2008;46:A1-A43.

9. Connolly BA, Sanford DG, Chiluwal AK, Healey SE, Peters DE, Dimare MT, et al. Dipeptide boronic acid inhibitors of dipeptidyl peptidase IV: determinants of potency and in vivo efficacy and safety. J Med Chem 2008;51:6005-13.

10. Allen M, Heinzmann A, Noguchi E, Abecasis G, Broxholme J, Ponting $\mathrm{CP}$, et al. Positional cloning of a novel gene influencing asthma from chromosome $2 q 14$. Nat Genet 2003;35:258-63.

11. Deacon CF, Carr RD, Holst JJ. DPP4 inhibitor therapy: new directions in the treatment of type 2 diabetes. Front Biosci 2008;13:1780-94.

12. Drucker DJ. The role of gut hormones in glucose homeostasis. J Clin Invest 2007;117:24-32.

13. Amori RE, Lau J, Pittas AG. Efficacy and safety of incretin therapy in type 2 diabetes: systematic review and metaanalysis. J Am Med Assoc 2007;298:194-206.

14. Richter B, Bandeira-Echtler E, Bergerhoff K, Lerch C. Emerging role of dipeptidyl peptidase-4 inhibitors in the management of type 2 diabetes. Vasc Health Risk Manag 2008;4:753-68.

15. Lambeir AM, Scharpé S, De Meester I. DPP4 inhibitors for diabetes - what next? Biochem Pharmacol 2008;76: 1637-43.
16. Focosi D, Kast RE, Metelli MR, Benedetti E, Galimberti S, Papineschi F, et al. Enhancement of hematopoietic stem cell engraftment by inhibition of CXCL12 proteolysis with sitagliptin, an oral dipeptidyl-peptidase IV inhibitor: a report in a case of delayed graft failure. Leuk Res 2009;33:178-204.

17. Jost MM, Lamerz J, Tammen H, Menzel C, De Meester I, Lambeir AM, et al. In vivo profiling of DPP4 inhibitors reveals alterations in collagen metabolism and accumulation of an amyloid peptide in rat plasma. Biochem Pharmacol 2009;77:228-37.

18. Tagore DM, Nolte WM, Neveu JM, Rangel R, GuzmanRojas L, Pasqualini R, et al. Peptidase substrates via global peptide profiling. Nat Chem Biol 2009;5:23-5.

19. Phang JM, Pandhare J, Liu Y. The metabolism of proline as microenvironmental stress substrate. J Nutr 2008; 138:2008S-15S.

20. Beesley CE, Young EP, Finnegan N, Jackson M, Mills K, Vellodi $A$, et al. Discovery of a new biomarker for the mucopolysaccaridoses (MPS), dipeptidyl peptidase IV (DPP-IV; CD26), by SELDI-TOF mass spectrometry. Mol Gen Metab 2009; doi:10.1016/j.ymgme.2008.12.002.

Ingrid De Meester*

Simon Scharpé

Anne-Marie Lambeir

Laboratory of Medical Biochemistry, Department of

Pharmaceutical Sciences, University of Antwerp,

Antwerp, Belgium

*Corresponding author: Ingrid De Meester, Laboratory of Medical Biochemistry, Department of Pharmaceutical Sciences, University of Antwerp, Universiteitsplein 1, 2610 Antwerp, Belgium Phone: +32-3-8202727, Fax: +32-3-8202734,

E-mail: ingrid.demeester@ua.ac.be 